\title{
FAMILIES OF SETS ON A FINITE SET
}

\author{
C. J. ASHMAN and V. FICKER \\ (Received 28 July 1982; revised 9 February 1983 ) \\ Communicated by W. D. Wallis
}

\begin{abstract}
V. Krishnamurthy has shown that on a finite set $X$ all topologies can be mapped into a certain set of matrices of zeros and ones. In this paper it is shown that all lattices, algebras and rings on a finite set $X$ can be mapped onto particular sets of matrices of zeros and ones.
\end{abstract}

1980 Mathematics subject classification (Amer. Math. Soc.): 28 A 05, 05 B 20.

In this paper our aim is twofold. Firstly, we consider families of subsets of a finite set with particular reference to lattices, rings, algebras and topologies. Secondly, we consider finite matrices of zeros and ones with various sets of properties and show the pairing of the matrices with the families of subsets considered in the earlier part.

Krishnamurthy [2] showed that on a finite set all topologies are mapped onto a certain set of matrices of zeros and ones. We establish similar mappings for lattices, rings and algebras.

Let $X$ denote a finite set. Let $n$ be a positive integer and $N=\{1,2, \ldots, n\}$. Then we shall assume that $X=\left\{x_{i}: i \in N\right\}$. On finite sets the operations of arbitrary or countable unions (intersections) collapse into finite unions (intersections). Thus a topology $\mathscr{T}$ is a family of subsets of $X$ such that $\varnothing \in \mathcal{T}, X \in \mathcal{T}$, and $\mathscr{T}$ is closed under the operations of unions and intersections of sets.

We shall deal with certain subsets of the following set of set-operations:

$$
P_{0}=\left\{\cup,+,-, \ominus, \Delta, \cap, C, S_{b}, S_{r}\right\}
$$

where $U$ denotes the operation of union of sets, + denotes the disjoint union, denotes the difference, $\ominus$ denotes the proper difference, $\Delta$ denotes the symmetric

(C) 1984 Australian Mathematical Society $0263-6115 / 84 \$ A 2.00+0.00$ 
difference, $\cap$ denotes the intersection, $C$ denotes the complement, $S_{b}$ denotes the formation of subsets, and $S_{r}$ denotes the formation of supersets.

Let $\mathscr{F}$ be a family of subsets of $X$ such that $\varnothing \in \mathscr{F}$ and $\mathscr{F}$ be closed under the operation of intersection of sets. Given $x \in X$, we define $\mathscr{F}_{x}=\{A \in \mathscr{F}: x \in A\}$ and put $S(x)=\cap \mathscr{F}_{x}$ whenever $\mathscr{F}_{x} \neq \varnothing$, and $S(x)=\varnothing$ whenever $\mathscr{F}_{x}=\varnothing$.

The following proposition is now straightforward for any $\mathscr{F}$ with the above conditions.

Proposition 1. To each $x \in X$ there exists a unique set $S(x)$ such that:

(i) $S(x) \in \mathscr{F}$,

(ii) $S(x) \neq \varnothing$ implies $x \in S(x)$, and

(iii) $A \in \mathscr{F}$ and $x \in A$ imply $S(x) \subset A$.

We shall use the symbol $\delta(\mathscr{F})$ to denote the family of sets $\{S(x): x \in X\}$.

Below we give examples of families which satisfy the assumptions of the above proposition.

(a) A lattice of sets $巳$, which is a family of subsets of $X$ with the set of properties $\{\cap, \cup\}$ and $\varnothing \in \mathcal{L}$.

(b) A ring of sets $R$, which is a non-empty family of subsets of $X$ with any of the following sets of properties $\{\cup,-\},\{+,-\},\{\cup, \Theta\},\{\Delta, \cap\},\{-, \Delta\}$ or $\{U, \Delta\}$.

(c) An algebra of sets $Q$, which is a non-empty family of subsets of $X$ with any of the sets of properties $\{\cup, C\},\{\cap, C\}$ or $\{-, C\}$.

(d) A topology $\sigma$, which is a family of subsets of $X$ with the set of properties $\{\cup, \cap\}$ and such that $\varnothing \in \mathcal{T}$ and $X \in \mathcal{T}$.

(e) A non-empty family $\mathscr{F}$ of subsets of $X$ closed under the set operations from any of the following sets of operations $\{+, \cap\},\{-, \ominus\},\{-, \cap\},\{\Theta, \cap\}$, $\left\{\cup, S_{b}\right\},\left\{+, S_{b}\right\},\left\{-, S_{b}\right\},\left\{\Theta, S_{b}\right\},\left\{\Delta, S_{b}\right\},\left\{\cap, S_{b}\right\},\left\{C, S_{b}\right\},\left\{-, S_{r}\right\},\left\{\Theta, S_{r}\right\}$, $\left\{\Delta, S_{r}\right\},\left\{\cap, S_{r}\right\},\left\{C, S_{r}\right\},\left\{S_{b}, S_{r}\right\}$.

We shall deal mainly with 4 types of families of subsets of $X$, namely algebras, rings, topologies and lattices. The relationship between these types of families is indicated by the diagram below.

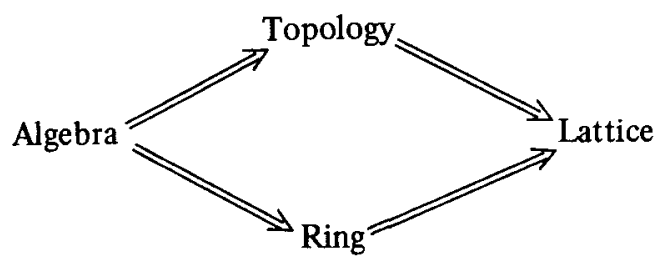


Proposition 2. Let $\mathcal{E}$ be a lattice of subsets of $X$ and $\mathscr{F}$ denote the following family

$$
\left\{\bigcup_{i \in I} S\left(x_{i}\right):\left(S\left(x_{i}\right) \in \delta(\mathcal{L})\right) \wedge(I \subset N)\right\} .
$$

Then $\mathcal{L}=\mathcal{F}$.

Proof. Let $A \in \mathcal{E}$ such that $A \neq \varnothing$. Then $A=\cup_{x \in A} S(x)$, since $x \in A$ implies $x \in S(x) \subset A$ hence $x \in \cup_{x \in A} S(x)$ and so $A \subset \cup_{x \in A} S(x)$. For $y \in$ $\cup_{x \in A} S(x)$ we have $y \in S(x)$ for some $x \in A$ hence $y \in S(x) \subset A$ implies $y \in A$. Thus $\cup_{x \in A} S(x) \subset A$. Therefore $A=\cup_{x \in A} S(x) \in \mathscr{F}$. Hence $\mathcal{C} \subset \mathscr{F}$. On the other hand, if $B \in \mathscr{F}$ and $B \neq \varnothing$ we have $B=\cup_{i \in I} S\left(x_{i}\right) \in \mathcal{Q}$ for some $I \subset N$. So $\mathscr{F} \subset \mathcal{E}$. Summarising, we have $\varrho=\mathscr{F}$.

Proposition 3. Let $R$ be a ring of subsets of $X$.

(i) If $S(x) \neq \varnothing$ then $y \in S(x)$ if and only if $S(y)=S(x)$.

(ii) For all $x, y \in X$ such that $x \neq y$, either $S(x) \cap S(y)=\varnothing$ or $S(x)=S(y)$. Further, if $\mathscr{F}=\left\{\cup_{i \in I} S\left(x_{i}\right):\left(S\left(x_{i}\right)\right) \in \mathcal{S}(\Re) \wedge(I \subset N)\right\}$ then $\Re=\mathscr{F}$.

Proof. (i) Assume that $S(x) \neq \varnothing$ for some $x \in X$. Further, suppose that $y \in S(x)$. By Proposition 1(iii), $S(y) \subset S(x)$. On the other hand, we show that $S(x) \subset S(y)$. This is equivalent to $S(x)-S(y)=\varnothing$. Assume on the contrary that either: (a) $S(x)-S(y) \neq \varnothing$ and $x \in S(x)-S(y)$; or (b) $S(x)-S(y) \neq$ $\varnothing$ and $x \notin S(x)-S(y)$. In case (a), we have $S(x)-S(y) \in \Re$, so by Proposition l(iii), $S(x) \subset S(x)-S(y)$. Since

$$
\varnothing \subset S(x) \cap S(y) \subset[S(x)-S(y)] \cap S(y)=\varnothing
$$

we have $S(x) \cap S(y)=\varnothing$. But this contradicts $S(x) \cap S(y) \neq \varnothing$ since $y \in S(x)$ hence $S(x) \in \mathscr{R}_{y}$ so $S(y) \neq \varnothing$ hence $y \in S(y)$ and $S(x) \cap S(y) \neq \varnothing$. In case (b) we assume that $S(x)-S(y)=\varnothing$ and $x \notin S(x)-S(y)$ then since $\varnothing \neq$ $S(x)-S(y) \subset S(x)$ implies $x \in S(x)$ and $x \in S(x)=[S(x)-S(y)] \cup[S(x)$ $\cap S(y)]$ implies $x \in S(x) \cap S(y) \subset S(y)$. Hence $S(x) \subset S(y)$ and so $S(x)-$ $S(y)=\varnothing$ which is a contradiction. Therefore, $S(x)-S(y)=\varnothing$ is true and so $S(x)=S(x) \cap S(y) \subset S(y)$.

Summarising, we have $y \in S(x)$ implies $S(y)=S(x)$. Now the second implication is easy since $S(x) \neq \varnothing$ implies $x \in S(x)=S(y)$ so $S(y) \neq \varnothing$ and $y \in$ $S(y)=S(x)$.

(ii) Let $A \in \mathscr{R}$ such that $A \neq \varnothing$.

We prove that if $S(x) \neq S(y)$ then $S(x) \cap S(y)=\varnothing$. It is sufficient to assume that $S(x) \neq \varnothing$ and $S(y) \neq \varnothing$. 
On the contrary, assume also that $S(x) \cap S(y) \neq \varnothing$. Then either $x \in S(x) \cap$ $S(y)$ or $x \notin S(x) \cap S(y)$. In the case $x \in S(x) \cap S(y)$ we have by (i) above, $x \in S(y)$ if and only if $S(x)=S(y)$, and this is a contradiction since we assumed $S(x) \neq S(y)$. In the case $x \notin S(x) \cap S(y)$ then $x \in S(x)-[S(x) \cap$ $S(y)] \in \mathcal{R}$, hence $S(x) \subset S(x)-[S(x) \cap S(y)]$ and then

$$
\begin{aligned}
\varnothing & \neq S(x) \cap S(y) \subset\{S(x)-[S(x) \cap S(y)]\} \cap S(y) \\
& =[S(x)-S(y)] \cap S(y)=\varnothing
\end{aligned}
$$

and this is a contradiction. Thus $S(x) \neq S(y)$ and $S(x) \cap S(y) \neq \varnothing$ leads in any case to a contradiction. So $S(x) \neq S(y)$ and $S(x) \cap S(y)=\varnothing$ is always true. Hence $S(x) \neq S(y)$ implies $S(x) \cap S(y)=\varnothing$. Hence for each $x \neq y$ the sets $S(x)$ and $S(y)$ are either identical or disjoint.

Since every ring of sets $\Re$ is also a lattice of sets we have by Proposition 2 that $\Re=\mathscr{F}$.

PROPOSITION 4. Let $\mathbb{Q}$ be an algebra of subsets of $X$. Then all the properties of Propositions 1, 2 and 3 are satisfied and $\cup_{x \in X} S(x)=X$.

Proof. Let $x \in X$. Then $X \in \mathbb{Q}_{x}=\{A \in \mathbb{Q}: x \in A\}$. Hence $\cap \mathbb{Q}_{x}=S(x) \subset X$ so $\cup_{x \in X} S(x) \subset X$.

On the other hand, if $x \in X$, there exists $A \in \mathbb{Q}, A \neq \varnothing$ such that $x \in A$, hence $S(x) \subset A$ so $x \in S(x) \subset \cup_{x \in X} S(x)$.

Thus $\cup_{x \in X} S(x)=X$.

Proposition 5. Let $\mathcal{T}$ be a topology of subsets of $X$. Then the family $\delta(\mathcal{T}) \cup$ $\{\varnothing, X\}$ forms a basis of neighbourhoods for the topology $\mathcal{T}$.

Proof. [2].

We shall denote by $\omega$ the set of all $n \times n$ matrices of zeros and ones. It is evident that $|\omega|=2^{n^{2}}$. Assume that $\left(a_{p q}\right)$ is an $n \times n$ matrix from $\omega$.

Certain matrices in $\omega$ may possess some combinations of properties from the list given below.

(a) $a_{l l}=0$ implies $a_{p l}=0=a_{l q}$ for each $p, q \in N$.

(b) $a_{I l}=1$ for each $l \in N$.

(c) For $i, j \in N$ and for each $k \in N, a_{i k}=1=a_{j k}$ implies $a_{i m}=a_{j m}$ for each $m \in N$.

(d) For $i, j \in N$ and each $k \in N, a_{j i}=1=a_{i k}$ implies $a_{j k}=1$. 
The following symbols will be used to denote the subsets of $\omega$ whose matrices satisfy the given combinations of properties from the above list:

$\lambda$ denotes the set of matrices with properties (a) and (d);

$\rho$ denotes the set of matrices with properties (a), (c) and (d);

$\alpha$ denotes the set of matrices with properties (b), (c) and (d);

$\tau$ denotes the set of matrices with properties (b) and (d).

Proposition 6. Let $\mathbf{F}$ be the collection of all families $\mathscr{F}$ of subsets of $X$ such that $\varnothing \in \mathscr{F}$ and $\mathscr{F}$ is closed under the formation of intersection of sets. Then there exists a mapping $T$ of $\mathscr{F}$ into $\omega$ where for each $\mathscr{F} \in \mathrm{F}$ the matrix $T(\mathscr{F})$ is defined as follows.

(i) If for $x_{i} \in X$ the set $S\left(x_{i}\right)=\varnothing$ then $a_{i j}=0$ for all $j$.

(ii) If $S\left(x_{i}\right) \neq \varnothing$ then $a_{i j}=1$ whenever $x_{j} \in S\left(x_{i}\right)$ and $a_{i j}=0$ whenever $x_{j} \notin S\left(x_{i}\right)$.

The uniqueness of elements in $\mathcal{S}(\mathscr{F})$ guarantees that $T$ is a mapping.

In what follows, Propositions 7 through 10 are associated respectively with Propositions 2 through 5.

Proposition 7. Let $\mathrm{L}$ be the collection of all lattices on $X$. Then there exists a one-to-one mapping $T$ of $L$ onto $\lambda$.

Proof. Define $T$ as in Proposition 6.

Then $T(\mathrm{~L}) \subset \omega$ where $T(\mathrm{~L})=\{T(\mathcal{L}): \varrho \in \mathrm{L}\}$.

Let $\mathcal{L}_{1}, \mathfrak{L}_{2} \in \mathrm{L}$ such that $\mathfrak{L}_{1} \neq \mathfrak{L}_{2}$.

Then there exists $A \in \mathscr{L}_{1}, A \neq \varnothing$ such that $A \notin \mathscr{L}_{2}$. Since $\varnothing \neq A \subset X$ assume that $|A|=k$. Now consider $S^{1}\left(x_{i}\right) \in \mathcal{S}\left(\mathcal{L}_{1}\right)$ for each $x_{i} \in A, i=1,2,3, \ldots, k$, and $S^{2}\left(x_{i}\right) \in \delta\left(\mathfrak{L}_{2}\right)$. Then there exists at least one $i_{0}$ such that $S^{1}\left(x_{i_{0}}\right) \neq S^{2}\left(x_{i_{0}}\right)$. On the contrary, assume that $S^{1}\left(x_{i}\right)=S^{2}\left(x_{i}\right)$ for each $i=1,2,3, \ldots, k$. Then $A=$ $\cup_{x_{i} \in A} S^{1}\left(x_{i}\right)=\cup_{x_{i} \in A} S^{2}\left(x_{i}\right) \in \mathcal{L}_{2}$. This contradicts the assumption that $A \notin \mathcal{L}_{2}$. Thus there is at least one $i_{0}$ such that $S^{1}\left(x_{i_{0}}\right) \neq S^{2}\left(x_{i_{0}}\right)$. Hence $\delta\left(\mathfrak{Q}_{1}\right) \neq \delta\left(\mathfrak{L}_{2}\right)$. Now the $i_{0}$ th row of the matrix $T\left(\mathscr{L}_{1}\right)$ is different from the $i_{0}$ th row of the matrix $T\left(\mathfrak{L}_{2}\right)$ and so $T\left(\mathfrak{L}_{1}\right) \neq T\left(\mathfrak{L}_{2}\right)$. Thus $T$ is a one-to-one mapping of $L$ into $\omega$.

Next, we show that $T$ is a mapping into $\lambda$. Assume that for some $\mathcal{L} \in L, T(\mathcal{L})$ is such that $a_{l l}=0$. That is, $x_{l} \notin S\left(x_{l}\right)$. Then, by Proposition 1(ii), $S\left(x_{l}\right)=\varnothing$ and so $a_{l q}=0$ for each $q \in N$. Now, we prove also that $a_{p l}=0$ for each $p \in N$. On the contrary, suppose that $a_{p l}=1$ for some $p$. Since $a_{l l}=0$ we have $p \neq l$ so $x_{l} \in S\left(x_{p}\right)$. That is, $S\left(x_{p}\right) \neq \varnothing$ and then $S\left(x_{p}\right) \in \mathcal{L}_{x_{l}}=\left\{A \in \mathcal{L}: x_{l} \in A\right\}$ hence $S\left(x_{l}\right)=\cap \mathcal{L}_{x_{l}} \neq \varnothing$ and this is a contradiction with $S\left(x_{l}\right)=\varnothing$. Thus $a_{p l}=0$ for each $p \in N$. So $T(\mathcal{E})$ satisfies the condition (a). Now, let $T(\mathcal{E})$ be such that $a_{i k}=1=a_{k j}$. We wish to prove that $a_{i j}=1$. 
From $a_{i k}=1=a_{k j}$ we have that $x_{k} \in S\left(x_{i}\right)$ and $x_{j} \in S\left(x_{k}\right)$. Since $S\left(x_{i}\right) \neq \varnothing$ and $S\left(x_{k}\right) \neq \varnothing$ we have that $x_{i}, x_{k} \in S\left(x_{i}\right)$ and $x_{j}, x_{k} \in S\left(x_{k}\right)$. So $x_{k} \in S\left(x_{i}\right)$ $\cap S\left(x_{k}\right)$. Further $x_{j} \in S\left(x_{k}\right) \neq \varnothing$ implies that $S\left(x_{k}\right) \in \mathcal{E}_{x_{j}}$, hence $S\left(x_{j}\right) \neq \varnothing$ and so $x_{j} \in S\left(x_{j}\right)$. Now, either $x_{j} \in S\left(x_{i}\right) \cap S\left(x_{k}\right)$, and since $S\left(x_{i}\right) \cap S\left(x_{k}\right) \subset$ $S\left(x_{i}\right)$, we have $a_{i j}=1$, or $x_{j} \notin S\left(x_{i}\right) \cap S\left(x_{k}\right)$, hence $x_{j} \in S\left(x_{k}\right)$ and $x_{j} \notin S\left(x_{i}\right)$. Now, since $x_{k} \in S\left(x_{k}\right) \cap S\left(x_{i}\right) \in \mathcal{L}$, so $S\left(x_{i}\right) \cap S\left(x_{k}\right) \in \mathcal{L}_{x_{k}}$, thus $S\left(x_{k}\right) \subset$ $S\left(x_{k}\right) \cap S\left(x_{i}\right)$, which is a contradiction since $S\left(x_{k}\right) \cap S\left(x_{i}\right)$ is a proper subset of $S\left(x_{k}\right)$, namely $x_{j} \in S\left(x_{k}\right)$ and $x_{j} \notin S\left(x_{i}\right)$, so finally $x_{j} \in S\left(x_{i}\right)$ and thus $a_{i j}=1$.

Now we show that $T$ is a mapping from $L$ onto $\lambda$. Assume that the matrix $\left(a_{i j}\right) \in \lambda$. We shall prove that there exists a lattice $\mathscr{L} \in \mathrm{L}$ such that $T(\mathcal{L})=\left(a_{i j}\right)$. Consider the $i$ th row of the matrix $\left(a_{i j}\right)$ and define the set $\Sigma\left(x_{i}\right)$ by

$$
\Sigma\left(x_{i}\right)=\left\{x_{j} \in X: a_{i j}=1\right\}
$$

and put $\Sigma\left(x_{i}\right) \equiv \varnothing$ whenever $a_{i j}=0$ for each $j \in N$. Further, define two families of sets $\mathscr{Q}=\left\{\Sigma\left(x_{i}\right): i \in N\right\}$ and $\mathcal{L}=\left\{\bigcup_{i \in I} \Sigma\left(x_{i}\right):(I \subset N) \wedge\left(\Sigma\left(x_{i}\right) \in \mathscr{D}\right)\right\}$.

We show that $\mathcal{L}$ is the required lattice. It is evident that $\mathcal{L}$ is closed under unions and contains the empty set.

To show that $\mathcal{L}$ is closed under intersections it is sufficient to show that for each pair $(i, j)$ the set $\Sigma\left(x_{i}\right) \cap \Sigma\left(x_{j}\right)$ is a finite union of sets from $\mathcal{D}$.

Either $\Sigma\left(x_{i}\right) \cap \Sigma\left(x_{j}\right)=\varnothing$ and then $\Sigma\left(x_{i}\right) \cap \Sigma\left(x_{j}\right) \in \mathcal{L}$, or $\Sigma\left(x_{i}\right) \cap \Sigma\left(x_{j}\right) \neq$ $\varnothing$, in which case we consider the following cases.

(i) $x_{i} \in \Sigma\left(x_{i}\right) \cap \Sigma\left(x_{j}\right)$, so $a_{i i}=1=a_{j i}$. Now, let $x_{k} \in \Sigma\left(x_{i}\right)$. Then $a_{i k}=1$, hence by $(d) a_{j k}=1$. That is, $x_{k} \in \Sigma\left(x_{j}\right)$. Hence $\Sigma\left(x_{i}\right) \subset \Sigma\left(x_{j}\right)$ and so $\Sigma\left(x_{i}\right) \cap$ $\Sigma\left(x_{j}\right)=\Sigma\left(x_{i}\right) \in \mathcal{L}$.

(ii) $x_{j} \in \Sigma\left(x_{i}\right) \cap \Sigma\left(x_{j}\right)$ leads to $\Sigma\left(x_{i}\right) \cap \Sigma\left(x_{j}\right)=\Sigma\left(x_{j}\right) \in \mathcal{L}$.

(iii) $x_{i} \notin \Sigma\left(x_{i}\right) \cap \Sigma\left(x_{j}\right)$ and $x_{j} \notin \Sigma\left(x_{i}\right) \cap \Sigma\left(x_{j}\right)$. Now, from the assumption, $\Sigma\left(x_{i}\right) \cap \Sigma\left(x_{j}\right) \neq \varnothing$ it follows that $\Sigma\left(x_{i}\right) \neq \varnothing$ and $\Sigma\left(x_{j}\right) \neq \varnothing$ and so $x_{i} \in \Sigma\left(x_{i}\right)$ and $x_{j} \in \Sigma\left(x_{j}\right)$. That is, $a_{i i}=1=a_{j j}$.

Since $x_{i} \notin \Sigma\left(x_{j}\right)$ and $x_{j} \notin \Sigma\left(x_{i}\right)$ we have $a_{i j}=0=a_{j i}$.

Consider any $x_{k} \in \Sigma\left(x_{i}\right) \cap \Sigma\left(x_{j}\right)$. That is, $a_{i k}=1=a_{j k}$. We shall prove that for $p \neq k$ and $p \in N$ we have $x_{p} \notin \Sigma\left(x_{i}\right) \cap \Sigma\left(x_{j}\right)$, that is, $a_{k p}=0$.

Assume that $x_{p} \notin \Sigma\left(x_{i}\right)$, that is, $a_{i p}=0$. Now, also $a_{k p}=0$, since on the contrary, if $a_{k p}=1$ then from (d), $a_{i p}=1$ (note $a_{i k}=1$ ), which is a contradiction, so $a_{k p}=0$. Similarly, from $x_{p} \notin \Sigma\left(x_{j}\right)$ we show that $a_{k p}=0$ whenever $a_{j p}=0$.

Combining the two parts we have that for each $r \neq k$, if $x_{r} \notin \Sigma\left(x_{i}\right) \cap \Sigma\left(x_{j}\right)$, then $a_{k r}=0$, thus $x_{r} \notin \Sigma\left(s_{k}\right)$. That is, $x_{r} \notin \Sigma\left(x_{i}\right) \cap \Sigma\left(x_{j}\right) \Rightarrow x_{r} \notin \Sigma\left(x_{k}\right)$, which is equivalent to $x_{r} \in \Sigma\left(x_{k}\right) \Rightarrow x_{r} \in \Sigma\left(x_{i}\right) \cap \Sigma\left(x_{j}\right)$ for $r \neq k$. Hence $\Sigma\left(x_{k}\right) \subset$ $\Sigma\left(x_{i}\right) \cap \Sigma\left(x_{j}\right)$, and so $\cup_{x_{k} \in \Sigma\left(x_{i}\right) \cap \Sigma\left(x_{j}\right)} \Sigma\left(x_{k}\right) \subset \Sigma\left(x_{i}\right) \cap \Sigma\left(x_{j}\right)$. Now, from $x_{k} \in$ 
$\Sigma\left(x_{i}\right) \cap \Sigma\left(x_{j}\right)$ we have $x_{k} \in \Sigma\left(x_{k}\right)$, hence

$$
\Sigma\left(x_{i}\right) \cap \Sigma\left(x_{j}\right) \subset \bigcup_{x_{k} \in \Sigma\left(x_{i}\right) \cap \Sigma\left(x_{j}\right)} \Sigma\left(x_{k}\right) .
$$

Finally, $\Sigma\left(x_{i}\right) \cap \Sigma\left(x_{j}\right)=\cup_{x_{k} \in \Sigma\left(x_{i}\right) \cap \Sigma\left(x_{j}\right)} \Sigma\left(x_{k}\right)$ provided that $x_{i}, x_{j} \notin \Sigma\left(x_{i}\right) \cap$ $\Sigma\left(x_{j}\right)$. Hence $\Sigma\left(x_{i}\right) \cap \Sigma\left(x_{j}\right) \in \mathcal{L}$.

Proposition 8. Let $\mathbf{R}$ be the collection of all rings of subsets of $X$. Then there exists a one-to-one mapping $T$ of $\mathrm{R}$ onto $\rho$.

Proof. Since each ring $\Re \in R$ is also a lattice, consider the restriction to $R$ of the mapping, say $T_{1}: L \rightarrow \lambda$ and denote this new mapping $T: R \rightarrow \lambda$. We shall show that $T$ is also onto $\rho$.

First, we prove that $T$ is a mapping into $\rho$. It is sufficient to show that for $\Re \in R$ the matrix $T(\Re)$ has property (c), since the other properties, namely (a) and (d) follow from Proposition 7. Consider $i, j \in N$ and the matrix $\left(a_{i j}\right)$ and assume that for each $i \neq j$ there exists a $k \in N$ such that $a_{i k}=1=a_{j k}$, that is, $x_{k} \in S\left(x_{i}\right)$ and $x_{k} \in S\left(x_{j}\right)$. Now, by Proposition 3(i), we have $S\left(x_{i}\right)=S\left(x_{k}\right)$ and $S\left(x_{k}\right)=S\left(x_{j}\right)$. Hence, $S\left(x_{i}\right)=S\left(x_{j}\right)$ or $a_{i m}=a_{j m}$ for each $m \in N$.

To prove that $T$ is a mapping of $\mathbf{R}$ onto $\rho$, consider a matrix $\left(a_{i j}\right) \in \rho$ and proceed as in Proposition 7.

Proposition 9. Let $\mathrm{A}$ be the collection of all algebras of subsets of $X$. Then there exists a one-to-one mapping $T$ of A onto $\alpha$.

Proof. Since each algebra $\mathcal{Q} \in \mathbf{A}$ is also a ring, consider the mapping $T: A \rightarrow \rho$ which is the restriction of the mapping from $R$ onto $\rho$. We shall show that $T$ is also onto $\alpha$.

First, we prove that $T$ is a mapping into $\alpha$. Now it is sufficient to show that the matrix $T(\mathbb{Q})$ has the property (b), since all the other properties follow from Propositions 7 and 8. Consider an arbitrary element $x_{i} \in X$. By Proposition 4, $X=\cup_{k \in N} S\left(x_{k}\right)$ and each $S\left(x_{k}\right) \neq \varnothing$ so $x_{i} \in S\left(x_{i}\right)$, thus $a_{i i}=1$ for each $i \in N$.

Next, we show that $T$ is a mapping from $A$ onto $\alpha$. Consider a matrix $\left(a_{i j}\right) \in \alpha$ and proceed as in Proposition 7. We define the families of sets

$$
\mathscr{D}=\left\{\Sigma\left(x_{i}\right): i \in N\right\} \quad \text { and } \quad \mathcal{Q}=\left\{\bigcup_{i \in I} \Sigma\left(x_{i}\right):\left(\Sigma\left(x_{i}\right) \in \mathscr{D}\right) \wedge(I \subset N)\right\} .
$$

We shall prove that $\mathscr{Q}$ is an algebra of subsets of $X$. We already know that $\mathscr{Q}$ is a ring of subsets of $X$. It is sufficient to prove that $X \in \mathbb{Q}$. By property (b), 
$x_{i} \in \Sigma\left(x_{i}\right)$ for each $I \in N$ so

$$
X=\bigcup_{i \in N}\left\{x_{i}\right\}=\bigcup_{i \in N} \Sigma\left(x_{i}\right) \in \mathbb{Q} .
$$

Proposition 10. Let $\mathrm{T}$ be the collection of all topologies of subsets of $X$. Then there exists a one-to-one mapping $T$ of $\mathrm{T}$ onto $\tau$.

Proof. See [2].

Krishnamurthy [2] enumerated the topologies on finite sets by counting certain matrices of zeros and ones. We can also enumerate lattices, rings and algebras on a given finite set by generating and counting the relevant matrices.

Evans, Harary and Lynn [1] developed a recurrence relation involving Stirling numbers of the second kind to find the number of topologies on a finite set and from this result Levinson and Silverman [3] derived an asymptotic formula. Similar recurrence relations can now be found for the number of algebras, rings and lattices on a finite set.

By considering the matrices as representative of the various families of subsets, it is possible to develop alternative sets of axioms, which determine whether or not a given matrix of zeros and ones represents a lattice, topology, algebra or ring of subsets.

\section{References}

[1] J. W. Evans, F. Harary and M. S. Lynn, 'On the computer enumeration of finite topologies', Comm. ACM 10 (1967), 295-298.

[2] V. Krishnamurthy, 'On the number of topologies on a finite set', Amer. Math. Monthly 73 (1966), 154-157.

[3] H. Levinson and R. Silverman, 'Topologies on finite sets II', Proc. Tenth S-E Conf. Comb. Graph Theory Comput. (1979), pp. 699-712.

\section{Department of Mathematics}

University of Newcastle

Newcastle, N.S.W.

Australia 2308 